\title{
Toward Low-Energy Spark-Plasma Sintering of Hot-Deformed Nd-Fe-B Magnets
}

\author{
Matic Korent, ${ }^{1,2, *}$, Marko Soderznik ${ }^{1,3}$, Urska Rocnik ${ }^{1}$, Sandra Drev ${ }^{4}$, Kristina Zuzek Rozman ${ }^{1,2}$, \\ Saso Sturm ${ }^{1,2}$, Spomenka Kobe ${ }^{1,2}$, Kristina Zagar Soderznik ${ }^{1}$ \\ ${ }^{1}$ Department for Nanostructured Materials, Jozef Stefan Institute, Ljubljana, Slovenia \\ ${ }^{2}$ Jozef Stefan International Postgraduate School, IPS, Ljubljana, Slovenia \\ ${ }^{3}$ Laboratory for Tribology and Interface Nanotechnology (TINT), Faculty for Mechanical Engineering, University of Ljubljana, Ljubljana, \\ Slovenia \\ ${ }^{4}$ Center for Electron Microscopy and Microanalysis, Jozef Stefan Institute, Ljubljana, Slovenia
}

\section{Email address:}

matic.korent@ijs.si (M. Korent), marko.soderznik@gmail.com (M. Soderznik), urska.rocnik@gmail.com (U. Rocnik),

sandra.drev@ijs.si (S. Drev), tina.zuzek@ijs.si (K. Z. Rozman), saso.sturm@ijs.si (S. Sturm), spomenka.kobe@ijs.si (S. Kobe),

kristina.zagar@ijs.si (K. Z. Soderznik)

${ }^{*}$ Corresponding author

\section{To cite this article:}

Matic Korent, Marko Soderznik, Urska Rocnik, Sandra Drev, Kristina Zuzek Rozman, Saso Sturm, Spomenka Kobe, Kristina Zagar Soderznik. Toward Low-Energy Spark-Plasma Sintering of Hot-Deformed Nd-Fe-B Magnets. International Journal of Materials Science and Applications. Vol. 10, No. 5, 2021, pp. 98-107. doi: 10.11648/j.ijmsa.20211005.12

Received: August 24, 2021; Accepted: September 13, 2021; Published: September 29, 2021

\begin{abstract}
High-coercivity Nd-Fe-B permanent magnets are key materials for producing electrical components on the macroand nanoscale. We present a newly developed, economically efficient method for processing Nd-Fe-B magnets based on spark-plasma sintering (SPS) that makes it possible to retain the technologically essential properties of the magnet, but by consuming about $30 \%$ less energy compared to the conventional SPS process. A magnet with an anisotropic microstructure was fabricated from MQU-F commercial ribbons with a low energy consumption $(0.37 \mathrm{MJ})$ during the deformation process and compared to a conventionally prepared hot-deformed magnet that consumed three-times more energy (1.2 MJ). Both magnets were post-annealed at $650^{\circ} \mathrm{C}$ for $120 \mathrm{~min}$ in a vacuum. After the post-annealing process, the low-energy processing (LEP) hot-deformed magnet exhibited a coercivity of $1327 \mathrm{kAm}^{-1}$, and a remanent magnetization of $1.27 \mathrm{~T}$. In comparison, the high-energy processing (HEP) hot-deformed magnet had a coercivity of $1337 \mathrm{kAm}^{-1}$ and a remanent magnetization of $1.31 \mathrm{~T}$. A complete microstructural characterization and detailed statistical analyses revealed a better texture orientation for the HEP hot-deformed magnet processed with the larger energy consumption. This texture is the main reason for the difference in the remanent magnetization between the two hot-deformed magnets. The results show that although the LEP hot-deformed magnet was processed with three-times less energy than in a typical hot-deformation process, the maximum energy product is only $8 \%$ lower than that of a HEP hot-deformed magnet.
\end{abstract}

Keywords: Hot-Deformed Nd-Fe-B Magnets, Low-Energy Processing Hot-Deformation Process, Spark-Plasma Sintering, Statistical Analyses, Electron Microscopy

\section{Introduction}

Discovered in 1984 [1, 2], Nd-Fe-B magnets are those with the highest maximum energy product $(B H)_{\max }$, more than $400 \mathrm{kJm}^{-3}$, which is close to the theoretical limit of 512 $\mathrm{kJm}^{-3}[3-7]$. Thus, they are in a very high demand for major applications in electric and hybrid vehicles, traction motors, and wind turbines $[8,9] . \mathrm{Nd}_{2} \mathrm{Fe}_{14} \mathrm{~B}$ has a tetragonal crystal structure with a $\mathrm{P}_{2} / \mathrm{mnm}$ space group [10]. To achieve a large remanent magnetization $\left(\mu_{0} M_{r}\right)$ and consequently a large value of $(B H)_{\max }$ for a magnet with such a composition, a high degree of (001) texture must be introduced [11, 12]. In addition, a high-volume percentage of the $\mathrm{Nd}_{2} \mathrm{Fe}_{14} \mathrm{~B}$ phase, a low oxygen content, a small amount of non-ferromagnetic 
material and a small crystallite size, close to the single-domain size $(\sim 250 \mathrm{~nm})$, is desirable [13]. Besides the sintering of magnetically pre-aligned particles, the hot-deformation (HD) process is a method in which the grain-boundary migration and sliding of crystal planes are induced by plastic deformation [11, 12, 14]. In 1985, Lee et al. and Tang et al. [15-17] produced HD magnets having an ultrafine microstructure with an average grain size from 300 $\mathrm{nm}$ to $400 \mathrm{~nm}$ from melt-spun Nd-Fe-B ribbons. The rapidly solidified powder with randomly orientated nanocrystals was transformed into textured, closely packed, plate-like grains with a high degree of $c$-axis alignment along the pressing direction during the HD process, resulting in a large $\mu_{0} M_{r}[18$, 19]. Recently, Tang et al. prepared ribbons with a small addition of $\mathrm{Nb}(0.29 \mathrm{wt} . \%)$ and performed the HD process at $850^{\circ} \mathrm{C}$ to obtain a $\mathrm{HD}$ magnet with a $75 \%$ height reduction and a $\mu_{0} M_{r}$ of $1.45 \mathrm{~T}$, which is comparable to the values of sintered magnets $[11,20]$.

The coercivity $\left(\mu_{0} H_{c}\right)$ of HD magnets greatly depends on the homogenous distribution of Nd-rich phases around the $\mathrm{Nd}_{2} \mathrm{Fe}_{14} \mathrm{~B}$ grains [21]. Sufficient magnetic isolation effectively enhances the value of the coercivity with a reduction of the exchange coupling between the $\mathrm{Nd}_{2} \mathrm{Fe}_{14} \mathrm{~B}$ grains $[21,22]$. Furthermore, it acts as a barrier to prevent domain-wall motion during the magnetization-reversal process $[12,23,24]$. However, detailed microstructure investigations by Lewis et al. and Liu et al. showed that the Nd-rich intergranular phase contains up to 50 at.\% ferromagnetic elements (pure metals of Fe and Co), which suggests the ferromagnetic nature of the isolation phase and consequently the exchange coupling between $\mathrm{Nd}_{2} \mathrm{Fe}_{14} \mathrm{~B}$ grains $[21,25,26]$. Consequently, Liu et al. showed that heavy-rare-earth-free HD magnets could have a $\mu_{0} H_{c}$ only up to $1430 \mathrm{kAm}^{-1}$ at room temperature (RT) [21].

In addition to the microstructure characteristics, processing conditions have a significant impact on the magnetic properties of HD magnets. According to Hioki et al., both the reduction ratio and hot-deformation temperature define the final $\mu_{0} M_{r}, \mu_{0} H_{c}$, and $(B H)_{\max }$ [27]. The value of $\mu_{0} M_{r}$ is closely related to the reduction ratio, as established by Korent et al. [28]. The increase in $\mu_{0} M_{r}$ is proportional to the reduction ratio, due to the higher degree of texture and a predominant c-plane growth of the $\mathrm{Nd}_{2} \mathrm{Fe}_{14} \mathrm{~B}$ grains [12, 27] . Additionally, the working temperature is important in terms of grain growth as it causes a deterioration of the magnetic properties [27].

Typically, HD magnets are hot-pressed (HP) (Figure 1a) with heating rates that are rather low, i.e., $5-10^{\circ} \mathrm{C} \mathrm{min}^{-1}$ [29]. Usually, the system includes a coil and the heating is based on induction or electrical resistance [30]. Another option for similar processing is the spark-plasma sintering (SPS) method (Figure 1b), in which the densification process is simultaneously applied by a uniaxial pressure and a large pulse of direct current (DC) [29]. The application of a mechanical force is like that in the HP process. Meanwhile, the heating rates, up to $1000^{\circ} \mathrm{C} \mathrm{min}^{-1}$, are much higher in comparison to HP magnets [29]. Efficient and rapid densification in the SPS method occurs through a combination of Joule heating, the action of spark discharge, the plastic-deformation effect, and the diffusion of material [31]. However, according to Chawake et al., Joule heating is the primary source of heat during the densification of metal powders [32]. The heating occurs due to the electrical resistance, which is most significant during the contact between the particles [32]. Consequently, the SPS process enables ultra-fast sintering at low temperatures and short holding times to achieve full material density, which preserves the magnetic properties and reduces the power consumption of the process itself $[33,34]$.
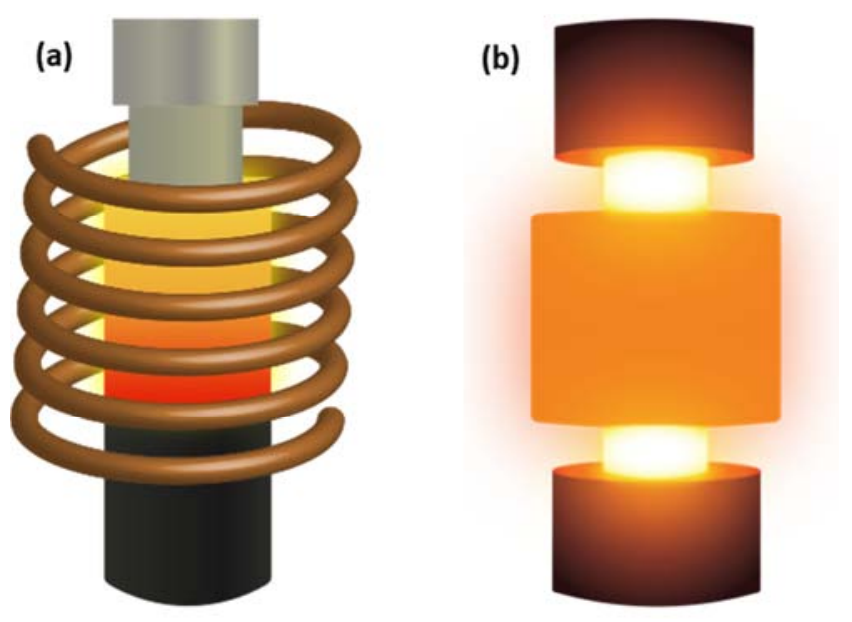

Figure 1. Schematic of (a) HP method and (b) SPS method.

In this work, the SPS method was used to fabricate a fully dense $\mathrm{HD} \mathrm{Nd}-\mathrm{Fe}-\mathrm{B}$ magnet with a high deformation ratio based on the low-energy processing (LEP) SPS method. The microstructure and its influence on the final magnetic properties were studied and the results obtained were compared to the microstructure of a HD magnet produced using the high-energy processing (HEP) SPS method.

\section{Experimental}

Commercial (MQU-F) Nd-Fe-B melt-spun ribbons with the nominal composition $\mathrm{Nd}_{30.1} \mathrm{Pr}_{0.4} \mathrm{Fe}_{62.0} \mathrm{Co}_{6.1} \mathrm{Ga}_{0.5} \mathrm{~B}_{0.9}$ (wt.\%) were used for the HD process. MQU-F ribbons were first HP into a fully dense magnet using a SPS furnace (Fuji Electronic Industrial Dr Sinter SPS Syntex 3000). The magnet was compacted in a 10 -mm graphite mold at $675^{\circ} \mathrm{C}$ under a uniaxially applied pressure of $50 \mathrm{MPa}$ in a vacuum $(\approx$ $5 \times 10^{-2}$ mbar $)$. The SPS temperature was controlled by a K-type thermocouple inserted into the graphite mold at the level of the sample. The thickness of the graphite wall splitting the thermocouple from the sample was minimal $(\approx 1$ $\mathrm{mm})$. A 12:2 an on-off DC pulse sequence was applied to heat the sample. After the sintering process was finished, the pressure was immediately released and the mold with the sample was cooled to RT with water circulating through the system. The density of the HP magnet was determined using a bulk-density measurement system (Densitec, Exelia AG, Switzerland). 
The HP magnets were subsequently HD in a $20-\mathrm{mm}$ graphite mold at two different maximum SPS temperatures in a vacuum. The temperatures below are the nominal temperatures and serve as an SPS processing parameter to determine the energy consumption through the deformation process. The SPS settings and measurement were equal to the HP process described in the previous paragraph. For processing the LEP HD magnet, the SPS temperature was set to a nominal $350^{\circ} \mathrm{C}$, and for the HEP $\mathrm{HD}$ magnet, at a nominal $680^{\circ} \mathrm{C}$. In the first case (LEP HD magnet) the heating rate from RT to the nominal temperature of $300^{\circ} \mathrm{C}$ was $100^{\circ} \mathrm{Cmin}^{-1}$, followed by a lower heating rate to the final temperature to minimize the overshoot. In the last minute, the temperature was maintained at the target temperature. The higher pressure was initially applied at a nominal temperature of $250^{\circ} \mathrm{C}$, and within $3.5 \mathrm{~min}$ it increased to a maximum pressure of $90 \mathrm{MPa}$. After the target temperature was reached, the sintering process was stopped immediately and the pressure was released. Meanwhile, in the second case (HEP HD magnet), the heating rate from RT to $600^{\circ} \mathrm{C}$ was $75^{\circ} \mathrm{Cmin}^{-1}$, followed by a lower heating rate to the final temperature to prevent the overshoot. In the last minute, the temperature was maintained at the target temperature, and the maximum pressure of $90 \mathrm{MPa}$ was applied during this holding time. As prepared, the HD magnets were subjected to a post-annealing process at $650^{\circ} \mathrm{C}$ for $120 \mathrm{~min}$ in a vacuum. The magnetic properties of both HP spark-plasma-sintered HD magnets before and after annealing were measured with a permeameter (Steingroever EP2) at RT. The deformation ratio of the LEP HD magnet was $71 \%$, and that of the HEP HD magnet, $75 \%$. The deformation ratio (\%) is defined as:

$$
\mathrm{DR}=\left(\left(\mathrm{h}_{\mathrm{HP}}-\mathrm{h}_{\mathrm{HD}}\right) / \mathrm{h}_{\mathrm{HP}}\right) \cdot 100 \%
$$

where DR represents the deformation ratio (\%) and $h_{H P}, h_{H D}$ are the heights of the HP, HD magnet (mm).

The X-ray powder-diffraction data were collected from the sample's surface perpendicular to the pressing direction with a Malvern Panalytical Empyrean X-ray diffractometer (XRD) using monochromated $\mathrm{X}$-rays, produced by a $\mathrm{Cu}$ target tube $(0.15406 \mathrm{~nm})$. The scan range in Bragg angle was from $10^{\circ}$ to $90^{\circ}(2 \theta)$. The XRD data were analyzed with HighScore Plus XRD Analysis Software. The microstructural characterization using scanning electron microscopy (SEM) was performed to study the influence of the SPS temperature and pressure on the HD process. The samples were cut into smaller square blocks, and the surface parallel to the pressing direction was metallographically polished. After polishing, the surface was chemically etched with 3-M Cyphos solution for $10 \mathrm{~s}$ and then intensively washed with ethanol. For the SEM microstructural characterization, samples were analyzed using a high-resolution, field-emission-gun SEM (FEG-SEM) Thermo Fisher, Verios G4 HP. The acquired images were statistically processed using the ImageTool program to determine the difference in the grain size and aspect ratio, as measured between the long and short axes of the anisotropic $\mathrm{Nd}_{2} \mathrm{Fe}_{14} \mathrm{~B}$ grains of the HD magnets. Detailed microstructural investigations of the samples were performed with transmission electron microscopy (TEM) using a 200-kV microscope (TEM; JEM-2100, Jeol, Japan). For the TEM bulk-sample investigations, the sample was cut into square blocks of $1.8 \times 1.8 \mathrm{~mm}^{2}$, perpendicular and parallel to the pressing direction. The blocks were fitted into $3-\mathrm{mm}$ brass cylinders using epoxy glue to improve the strength. The TEM specimen was ground to a thickness of $100 \mu \mathrm{m}$ and dimpled down to $15 \mu \mathrm{m}$ at the disc center (Dimple grinder, Gatan Inc., Warrendale PA, USA). The TEM specimen was finally ion-milled (PIPS, Precision Ion Polishing System, Gatan Inc., USA) using 3-kV $\mathrm{Ar}^{+}$ions at an incidence angle of $8^{\circ}$ until perforation.

\section{Results}

\subsection{LEP VS HEP HD Magnet}

Two differently prepared HD magnets could be distinguished based on the energy consumption for the deformation process. Figure 2 shows the electrical power (P) consumption during the SPS process of the LEP and HEP HD magnets. The energy consumption is the time integral of the electrical power, and its value was obtained from the area below the curves. During the deformation process of the LEP HD magnet, the consumed energy was $0.37 \mathrm{MJ}$, which is more than three-times less than the $1.2 \mathrm{MJ}$ consumed in the HEP HD process.

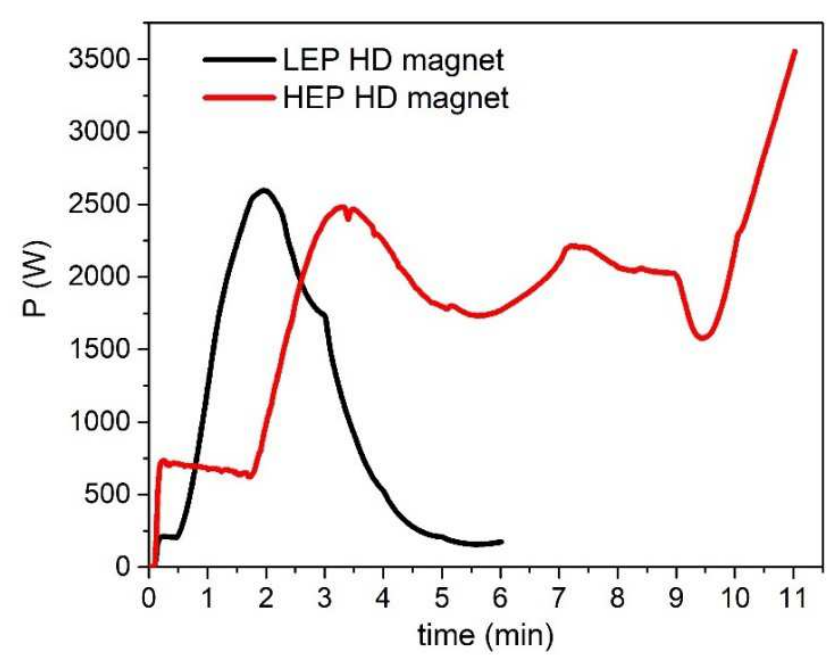

Figure 2. Electrical power $(P)$ consumption during the SPS process of LEP HD magnet (black curve) and HEP HD magnet (red curve). The area under the curve was obtained from a numerical integration $\int_{0}^{t_{e n d}} P \cdot d t$. The calculated energy consumptions are 0.37MJ for the LEP hot-deformation process and 1.2 MJ for HEP hot-deformation process.

\subsection{Magnetic Properties}

Figure 3 shows the demagnetization curves of the LEP and HEP HD magnets before and after the post-annealing treatment at $650^{\circ} \mathrm{C}$ for $120 \mathrm{~min}$. Note that the magnets were fully magnetized in a pulse magnetizer with $6 \mathrm{~T}$ before each measurement. 


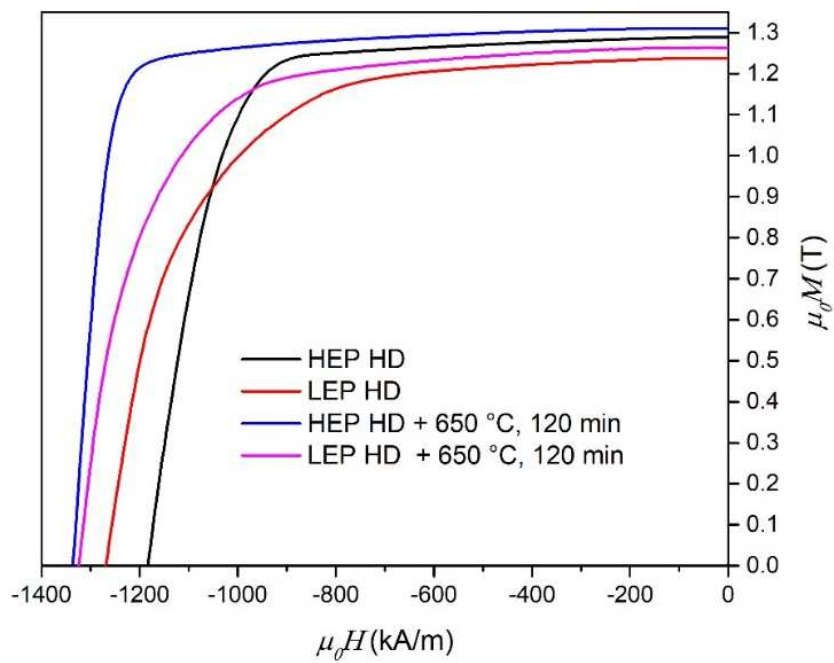

Figure 3. Demagnetization curves of LEP HD magnet (red curve) and HEP $H D$ magnet (black curve) and demagnetization curves of LEP (purple curve) and HEP (blue curve) HD magnets after the post-annealing process at $650^{\circ} \mathrm{C}$ for $120 \mathrm{~min}$.
The magnetic properties and the squareness (Q) of the demagnetization curves of each HD magnet are collected in Table 1 . The $\mu_{0} M_{r}$ of the LEP HD magnet was enhanced with the post-annealing treatment from $1.24 \mathrm{~T}$ to $1.27 \mathrm{~T}$, and $\mu_{0} H_{c}$ from $1271 \mathrm{kAm}^{-1}$ to $1327 \mathrm{kAm}^{-1}$. A similar improvement after post-annealing in $\mu_{0} M_{r}$, from $1.29 \mathrm{~T}$ to $1.31 \mathrm{~T}$, was also observed in the HEP HD magnet. Meanwhile, the enhancement in coercivity of the HEP HD magnet after the post-annealing process was more significant-from 1184 $\mathrm{kAm}^{-1}$ to $1337 \mathrm{kAm}^{-1}$. The squareness of the demagnetizing curves was also improved after additional heating. In the case of the LEP HD magnet, the squareness was enhanced from $70 \%$ to $77 \%$, and in the case of the HEP HD magnet from $82 \%$ to $91 \%$. The squareness is defined as:

$$
\mathrm{Q}=\mu_{0} \mathrm{H}_{\mathrm{k}} / \mu_{0} \mathrm{H}_{\mathrm{c}} \cdot 100 \%
$$

where $\mu_{0} H_{k}$ is a field value corresponding to the location of the knee point in the intrinsic curve at $90 \%$ of $\mu_{0} M_{r}$ [35].

Table 1. Magnetic properties $\left(\mu_{0} H_{c}, \mu_{0} M_{r},(B H)_{m a x}\right)$ and $Q$ of the demagnetizing curves of LEP, HEP HD magnets and of LEP, HEP HD magnets after the post-annealing process at $650^{\circ} \mathrm{C}$ for $120 \mathrm{~min}$.

\begin{tabular}{lllll}
\hline & LEP HD magnet & HEP HD magnet & LEP HD magnet $+\mathbf{6 5 0}^{\circ} \mathbf{C}, \mathbf{1 2 0}$ min & HEP HD magnet + 650 ${ }^{\circ} \mathbf{C}, \mathbf{1 2 0}$ min \\
\hline$\mu_{0} H_{c}\left(\mathrm{kAm}^{-1}\right)$ & 1271 & 1184 & 1325 & 1337 \\
$\mu_{0} M_{r}(T)$ & 1.24 & 1.29 & 1.27 & 1.31 \\
$(B H)_{\max }\left(\mathrm{kJm}^{-3}\right)$ & 295 & 320 & 310 & 335 \\
$Q(\%)$ & 70 & 82 & 77 & 91 \\
\hline
\end{tabular}

\subsection{Microstructural Characterization}

Figure 4 shows the XRD patterns of the LEP and HEP HD magnets after the post-annealing process. The analysis was performed on the surface perpendicular to the pressing direction. The characteristic peaks with directions (004), (105), (006), and (008) of the anisotropic microstructure are labelled in the chart.

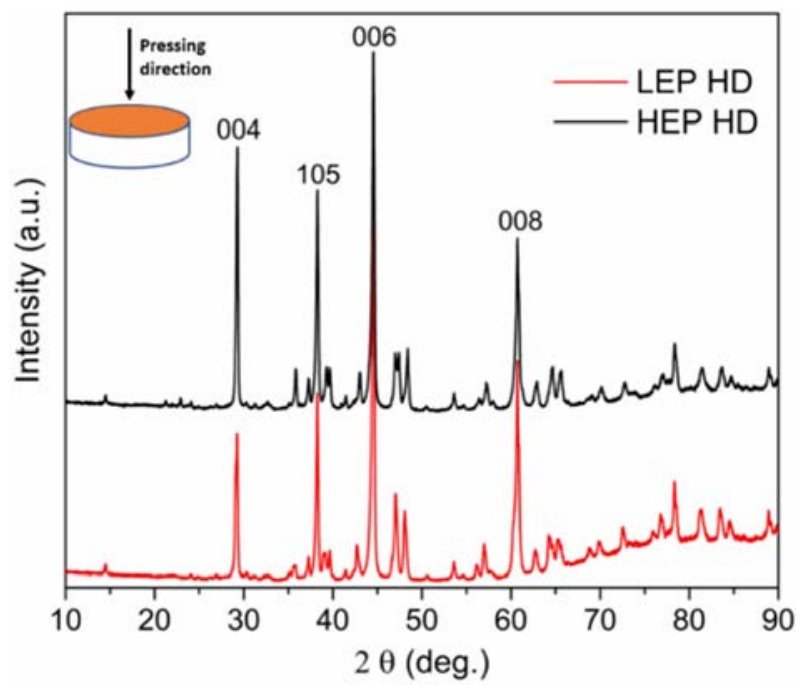

Figure 4. XRD patterns of LEP and HEP HD magnets after the post-annealing process at $650^{\circ} \mathrm{C}$ for $120 \mathrm{~min}$.

A backscattered-electron (BSE) SEM characterization was performed on the central part of the LEP and HEP HD magnets, to understand the influence of microstructure on the magnetic properties. Figure 5 shows cross-section images of (a) the LEP and (b) the HEP HD magnets after the post-annealing process, where the direction of the $c$-axis is the same as the pressure direction. Note, that the BSE SEM results of the LEP and HEP HD magnets prior to the annealing process gave identical results. In both images, coarse grains and grains with random orientations can be seen, especially in the LEP HD magnet. The main difference between those two magnets is in the microstructure texturing. A HEP HD magnet has a stronger $c$-axis alignment of the $\mathrm{Nd}_{2} \mathrm{Fe}_{14} \mathrm{~B}$ grains along the pressing direction than the LEP HD magnet, which will be confirmed later with more detailed information about the shape and orientation of the grains.

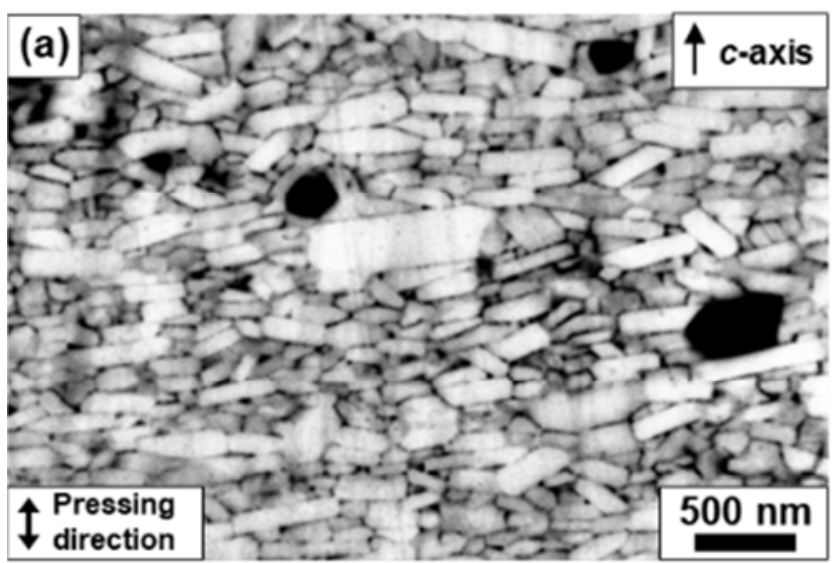




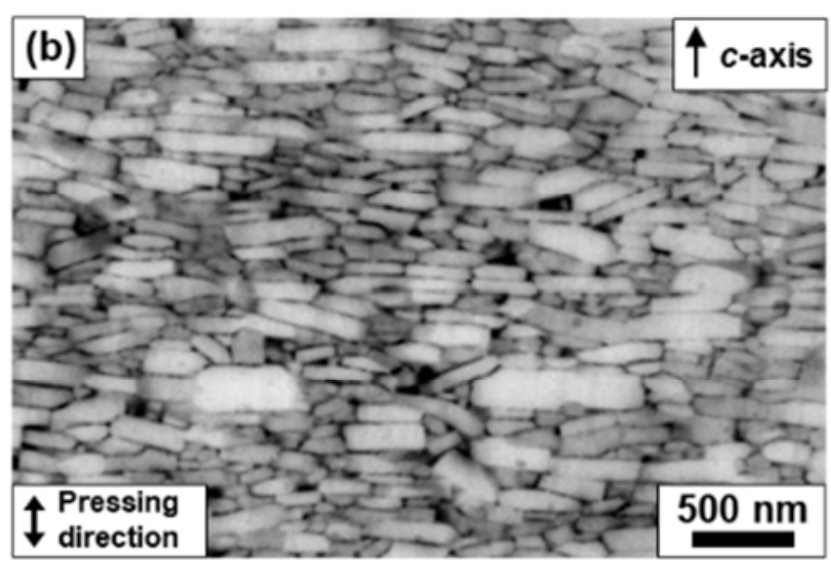

Figure 5. BSE SEM images of (a) LEP and (b) HEP HD magnet. (The SEM analyses were performed after the post-annealing process at $650^{\circ} \mathrm{C}$ for $120 \mathrm{~min}$.).
Based on the BSE SEM images, histograms and standard-distribution curves were obtained by counting the width and height of 200 characteristic grains that are not overlapped by others. Figure 6 shows the height and width distribution of the post-annealed LEP HD magnet $(a, b)$ and HEP HD magnet (c, d). The LEP HD magnet has a broader grain-height distribution with an average height of $63 \mathrm{~nm}$ (Figure 6a), compared to the HEP HD magnet with an average height of $67 \mathrm{~nm}$ (Figure $6 \mathrm{c}$ ). In the LEP HD magnet, $5 \%$ of the grains are larger than $160 \mathrm{~nm}$, whereas in the HEP HD magnet this is not the case. In contrast, the width-distribution curve of the HEP HD magnet (Figure 6d) is broader, with a more significant number of long grains and an average width of 348 $\mathrm{nm}$. Almost $100 \mathrm{~nm}$ lower, the average grain width of $252 \mathrm{~nm}$ is observed in the LEP HD magnet (Figure 6b).
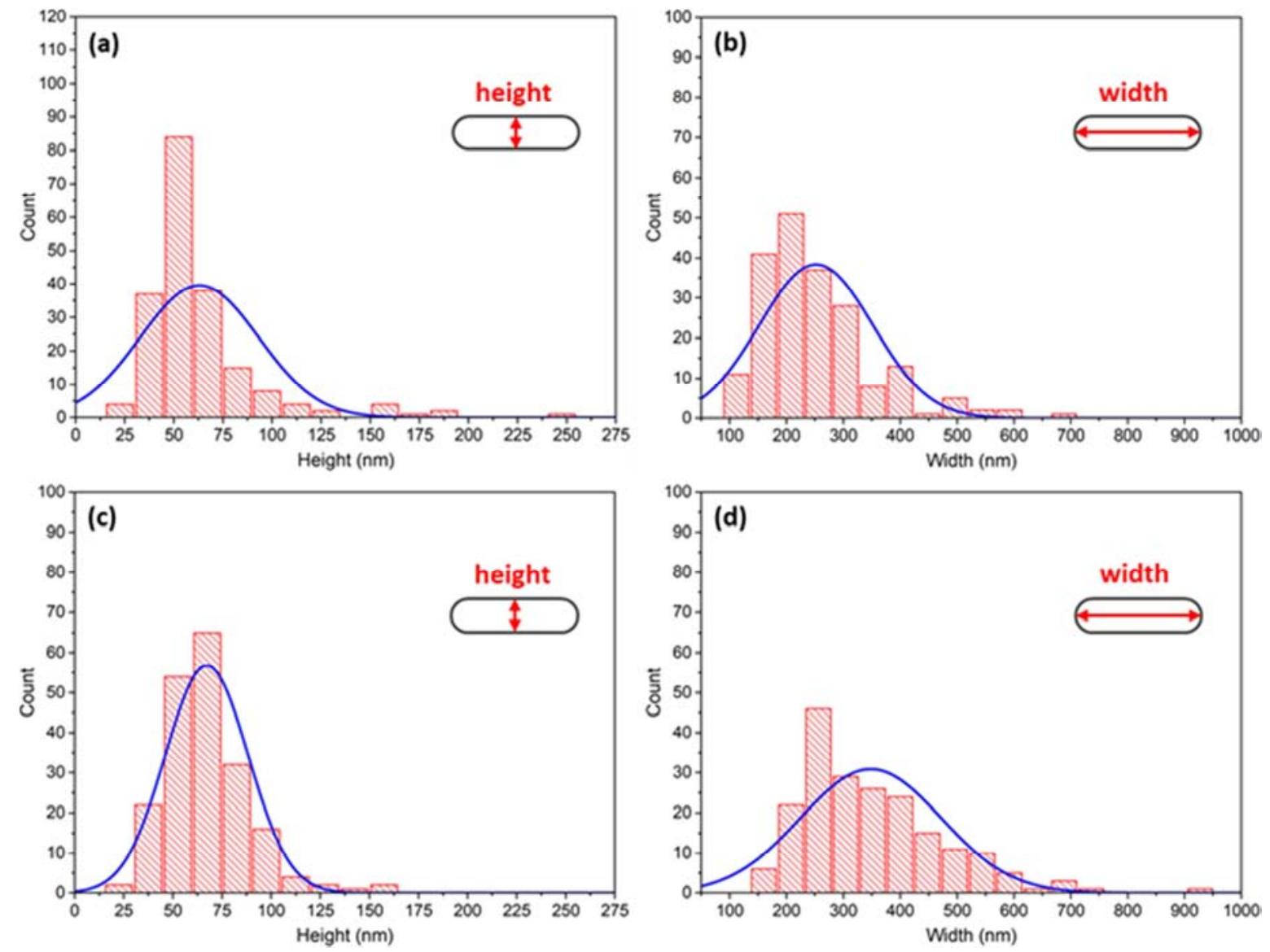

Figure 6. Statistical distribution of the height and width of 200 grains of the (a), (b) LEP and (c), (d) HEP HD magnets. (All statistical distributions were performed on the samples after the post-annealing process at $650^{\circ} \mathrm{C}$ for $120 \mathrm{~min}$.).

The aspect ratio (width/height) was calculated based on each grain's width and height. Figures 7 a, c presents the histograms and distributions of aspect ratios of 200 grains, where (a) illustrates the LEP and (c) the HEP HD magnets after post-annealing. The standard distribution curve of the analyzed grains from the HEP HD sample is broader, with an average aspect ratio of $\sim 5.3$. The LEP HD sample possesses grains with an aspect ratio between 2 and 6.5 and subsequently has a narrower distribution curve. The average aspect ratio is consequently lower, at $\sim 4.2$. Figure 7 also shows the angle between the pressing direction and the c-axes of the grains of (b) the LEP and (d) the HEP HD samples after post-annealing. In this case, the LEP HD sample's grain-distribution curve is broad, which means that many grains have an orientation out of the pressing direction. The average deviation angle is $8.3^{\circ}$. The HEP HD sample has a narrower distribution curve and a smaller number of grains with a high deviation angle from the pressing direction. The average deviation angle is $7.4^{\circ}$, which is almost $1^{\circ}$ lower than in the LEP HD sample. 

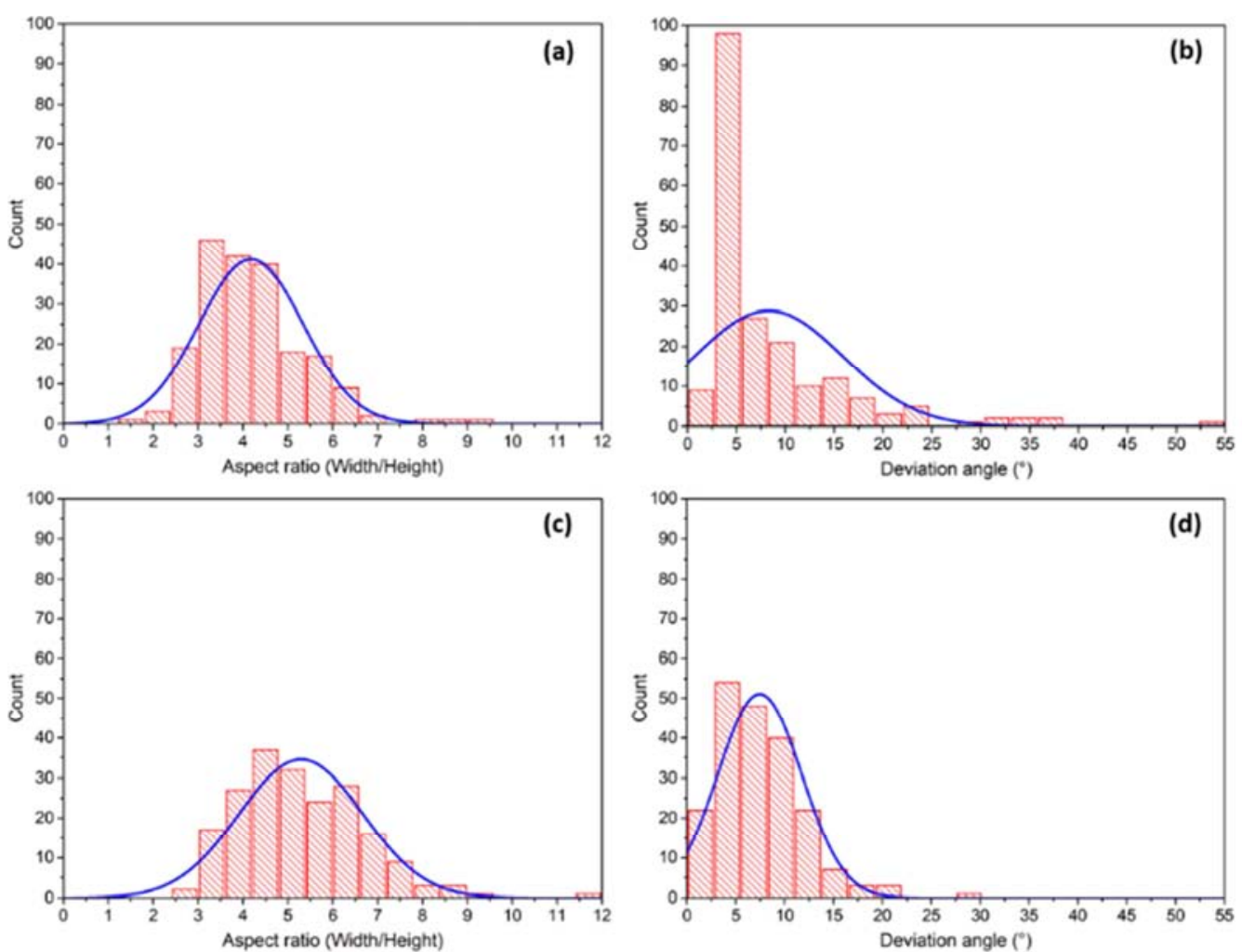

Figure 7. Statistical distribution of the aspect ratio (width/height) and deviation angle of 200 grains of (a), (b) LEP and (c), (d) HEP HD magnet. (All statistical distributions were performed after the post-annealing process at $650^{\circ} \mathrm{C}$ for $120 \mathrm{~min}$.).

Detailed TEM analysis (Figure 8) with selected-area electron-diffraction (SAED) patterns were performed parallel and perpendicular to the pressing direction of the LEP and HEP HD samples after the post-annealing process. Figures 8 $(\mathrm{a}, \mathrm{b})$ shows grains parallel to the $c$-axis. The low-magnification images show the size and shape of anisotropic grains that correspond to the SEM findings. Analysis of the grains determined a misalignment of $\pm 2-3$ degrees from the preferential plane. From a detailed analysis of the diffraction patterns, we confirmed that the preferred orientation of the grains is in the $(0-10)$ direction for both the LEP and HEP HD magnets.

Figures $8(\mathrm{c}, \mathrm{d})$ shows grains perpendicular to the $c$-axis. Low-magnification images of the LEP and HEP HD samples revealed the idiomorphic shape of the grains with a diameter of $0.1-0.8 \mu \mathrm{m}$. Analyses of the grains determined a misalignment of $\pm 1-2$ degrees from a preferential plane. Detailed analyses of the diffraction patterns show the preferential [001] plane of the grains that are related to the XRD analysis.
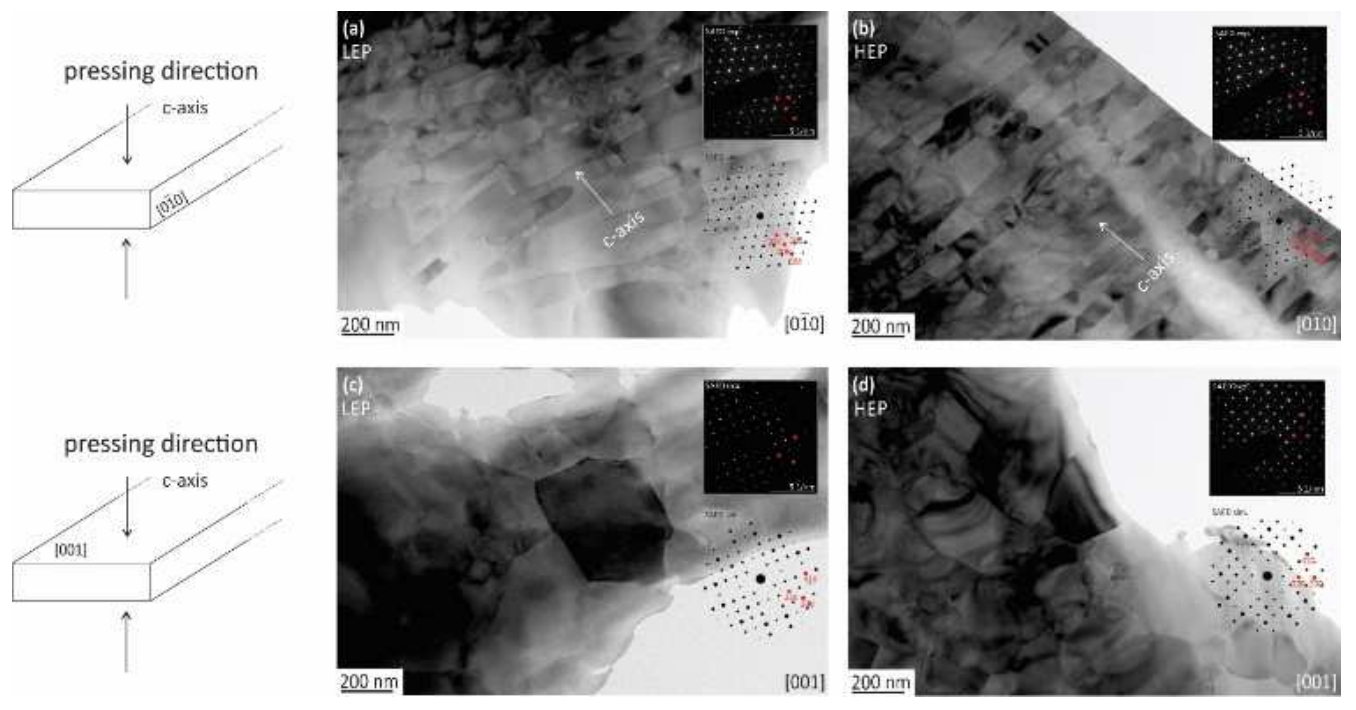

Figure 8. TEM analyses and SAED diffraction patterns (experimental and simulated) of (a) and (c) LEP HD and (b) and (d) HEP HD samples. The analyses were performed parallel and perpendicular to the c-axis (pressing direction). (TEM analyses were performed after the post-annealing process at $650^{\circ} \mathrm{C}$ for 120 min.). 


\section{Discussion}

The amount of energy transferred to the sample influences the temperature gradient in the hot-deformation process. Less energy should be reflected in a lower average temperature of the sample, which in the experiment is compensated by applying a specific pressure regime, so making the desired microstructure transformations possible.

The HEP HD magnet was processed at a nominal temperature comparable to previously set temperatures using the same technique and material. For example, S. Liu et al. produced a $\mathrm{HD}$ magnet at $700^{\circ} \mathrm{C}$ [36] and $\mathrm{Z}$. H. Hu et al. at $650-750^{\circ} \mathrm{C}$ [37]. We showed that the energy consumed during the deformation process is strongly related to the temperature regime, which indicates that a large amount of energy is consumed in a typical hot-deformation process. If the deformation temperature is lower, a large amount of energy can be saved. In our case during the LEP HD magnet's deformation process, the $0.37 \mathrm{MJ}$ of energy was consumed, while the HEP HD magnet's processing consumed three-times more energy. Moreover, assuming an average working power of $8 \mathrm{~kW}$ running for approximately $10 \mathrm{~min}$, a conventional hot-pressing machine consumes at least 10-times more energy for the hot-deformation process compared to LEP SPS.

We performed the XRD analysis perpendicular to the $c$-axis on the LEP and HEP HD magnets to confirm the $c$-axis crystallographic alignment. The XRD patterns of the LEP and HEP HD magnets after the post-annealing process show the enhanced intensity of the characteristic peaks (004), (006), (008), and (105), which indicates the development of the $c$-axis crystallographic alignment [38]. In parallel with the XRD, a TEM analysis was performed. The LEP and HEP HD samples' diffraction patterns coincide with the XRD results, which is an additional indicator of an anisotropic microstructure. From the perspective of magnetic properties, we achieved a high deformation of $\sim 71 \%$ in the LEP HD magnet and $\sim 75 \%$ in the HEP HD magnet. Hioki et al. showed that the $\mu_{0} M_{r}$ of a HD magnet changed between $70 \%$ and $75 \%$ of height reduction by only $0.02 \mathrm{~T}$, and it remained unchanged above $75 \%$ [27]. Consequently, we can assume that in our case, height reduction has a partial influence on $\mu_{0} M_{r}$ for HD magnets. However, despite the considerable height reduction in the HD magnets, there is still a visible difference of $0.05 \mathrm{~T}$ in $\mu_{0} M_{r}$ between the LEP HD magnet $(1.24 \mathrm{~T})$ and the HEP HD magnet $(1.29 \mathrm{~T})$, which can be attributed to the uniformity of the grain shape and orientation. The texture-formation mechanism for plate-like grain growth and alignment can be described by a solution-precipitation-creep model with the assistance of the Nd-rich phase [39-41]. Leonowicz et al. considered that liquid Nd-rich grain-boundary phases induce the sliding of grains under an applied shear stress, which results in the magnets' deformation and texture formation [42] To understand the origin of the difference in magnetic properties between the LEP and HEP HD magnets, the shape and orientation of particular grains in the microstructure were investigated. In the case of the HEP HD magnet, the plate-like grains have a relatively uniform shape and orientation of the $c$-axis parallel to the pressing direction. On the other hand, in the LEP HD magnet, the plate-like grains have a less-uniform shape and more scattered orientation. Castle et al. [43] reported that the reason for the uniform microstructure of the HD magnet is a more even temperature distribution across the powder flakes during the sintering. This implies that in the case of processing the LEP HD magnet, the temperature was uneven throughout the sample, which caused the non-uniformity in the final microstructure.

The above-described findings were supported by the counted data, where the results showed a higher aspect ratio between the width and the height of grains in the HEP HD magnet's microstructure. The average aspect ratio of $\sim 5.3$ was significantly higher than that for the LEP HD magnet ( 4.2). The aspect ratio is closely connected to the degree of deformation, which gives a partial explanation for the higher $\mu_{0} M_{r}$ and lowers the $\mu_{0} H_{c}$ of the HEP HD magnet $[12,40]$. To better understand the difference in $\mu_{0} M_{r}$, we also measured the angle between the grains' $c$-axes and the pressing direction for 200 grains from each HD magnet. Just as with the aspect ratio, the result showed a better grain orientation in the HEP HD magnet. The average deviation angle from the pressing direction is almost $1^{\circ}$ lower than for the LEP HD magnet, $8.3^{\circ}$. This indicates better grain orientation for the HEP HD magnet and, consequently, higher $\mu_{0} M_{r}$ and higher $(B H)_{\max }$ [40]. From this set of analyzed data, we can also infer the shape of the demagnetization curve. Diverse grain shapes and their more imperfect orientations have a crucial impact on the reduced squareness of the LEP HD magnet [35].

From the perspective of $\mu_{0} H_{c}$, the MQU-F ribbons were chosen for the deformation process due to their rich $\mathrm{Nd}$ content. There is $30.1 \mathrm{wt} . \% \mathrm{Nd}$ in the material composition, which is 4.1 wt.\% above the stoichiometric point. Liu et al. showed that within ribbons with a high $\mathrm{Nd}$ content, the fraction of Nd-rich phase increased along the grain boundaries [21]. Due to the increase in the Nd content and the decrease of the $\mathrm{Fe}$ and $\mathrm{Co}$ content in the grain boundaries, better isolation of the $\mathrm{Nd}_{2} \mathrm{Fe}_{14} \mathrm{~B}$ grains is established, which results in a higher value of $\mu_{0} H_{c}$ [21]. The value of $\mu_{0} H_{c}$ is higher for the LEP HD magnet, $1271 \mathrm{kAm}^{-1}$, compared to the value for the HEP HD magnet, $1184 \mathrm{kAm}^{-1}$. After the additional post-annealing step at $650^{\circ} \mathrm{C}$ for $120 \mathrm{~min}$, all the magnetic properties were enhanced, presumably due to the removal of internal stress and/or defects [44]. The $\mu_{0} M_{r}$ and $\mu_{0} H_{c}$ of the LEP HD magnet were increased by $0.3 \mathrm{~T}$ and $54 \mathrm{kAm}^{-1}$ compared to those of the HEP HD magnet, $0.2 \mathrm{~T}$ and $153 \mathrm{kAm}^{-1}$. According to Yin et al., the inherited crystalline orientation and improved texture promote the remanent magnetization after the post-annealing process [45].

Meanwhile, the post-annealing process's coercivity promotion is mainly related to the Nd-rich phase's enhanced distribution at the grain boundaries and the ribbon boundaries [45]. The LEP HD magnet's squareness was improved from $70 \%$ to $77 \%$, and the HEP HD magnet from 
$82 \%$ to $91 \%$. The microstructure homogeneity through the grain size distribution, grain shape, grain surface, and spatial distribution is improved by a post-annealing process, which is also the main reason for the enhanced squareness [35, 46]. Based on the statistical analyses, BSE SEM and TEM images, the HEP HD magnet's microstructure is more homogenous than the microstructure of the LEP HD magnet, which leads to a better squareness of the HEP HD magnet. Given the magnetic properties figure of merit improvement, both HD magnets exhibited $\sim 5 \%$ higher value of $(B H)_{\max }$ after the post-annealing process.

\section{Conclusions}

LEP and HEP HD magnets were processed using an SPS technique, which uses less energy than the typical hot-deformation process. The microstructural and magnetic properties of the LEP and HEP HD magnets were investigated, and a detailed analytical comparison was performed. Prior to the characterization, the post-annealing step of both HD magnets was performed to further enhance the magnetic properties. The LEP HD magnet had a just $0.04 \mathrm{~T}$ lower remanent magnetization than the HEP HD magnet $(1.31 \mathrm{~T})$. After post-annealing, both magnets' coercivities are similar: $1325 \mathrm{kAm}^{-1}$ for the LEP HD and $1337 \mathrm{kAm}^{-1}$ for the HEP HD. A minor deficiency in the magnetic properties for the LEP HD magnet was revealed by statistical analyses, which showed a better texture orientation for the HEP HD magnet. The results indicated a slightly lower remanent magnetization and reduced squareness for the LEP HD magnet.

A hot-deformed magnet with a significantly lower energy consumption can be produced if a proper fabrication method is applied. Further studies are underway to resolve the mechanism of texture formation during low-energy processing and to generate even better magnetic properties for the HD magnets.

\section{Acknowledgements}

This work was supported by projects ID PR-08336 and P2-0084 of the Slovenian Research Agency.

\section{References}

[1] M. Sagawa, K. Hiraga, H. Yamamoto, and Y. Matsuura, "Permanent magnet materials based on the rare earth-iron-boron tetragonal compounds (invited)," IEEE Trans. Magn., vol. 20, no. 5, pp. 1584-1589, 1984, doi: 10.1109/TMAG.1984.1063214.

[2] J. J. Croat, J. F. Herbst, R. W. Lee, and F. E. Pinkerton, "High-energy product Nd-Fe-B permanent magnets," Appl. Phys. Lett., vol. 44, no. 1, pp. 148-149, 1984, doi: $10.1063 / 1.94584$.

[3] Yanglong Hou and David J. Sellmyer, Magnetic nanomaterials: Fundamentals synthesis and applications. Wiley-VCH Verlag $\mathrm{GmbH}, 2017$.
[4] T. Zhang et al., "Hot-deformed Nd-Fe-B magnets fabricated by dynamic loading with a high maximum energy product," Intermetallics, vol. 73, pp. 67-71, 2016, doi: 10.1016/j.intermet.2016.04.001.

[5] T. Saito, M. Fujita, T. Kuji, K. Fukuoka, and Y. Syono, "The development of high performance Nd-Fe-Co-Ga-B die upset magnets," J. Appl. Phys., vol. 83, no. 11, pp. 6390-6392, 1998, doi: $10.1063 / 1.367522$.

[6] K. Hono and H. Sepehri-Amin, "Strategy for high-coercivity Nd-Fe-B magnets," Scr. Mater., vol. 67, no. 6, pp. 530-535, 2012, doi: 10.1016/j.scriptamat.2012.06.038.

[7] M. Yue, M. Tian, J. X. Zhang, D. T. Zhang, P. L. Niu, and F. Yang, "Microstructure and magnetic properties of anisotropic Nd-Fe-B magnets produced by spark plasma sintering technique," Mater. Sci. Eng. B Solid-State Mater. Adv. Technol., vol. 131, no. 1-3, pp. 18-21, 2006, doi: 10.1016/j.mseb.2005.11.010.

[8] U. M. R. Seelam et al., "Coercivity of the Nd-Fe-B hot-deformed magnets diffusion-processed with low melting temperature glass forming alloys," J. Magn. Magn. Mater., vol. 412, pp. 234-242, 2016, doi: 10.1016/j.jmmm.2016.04.005.

[9] M. Soderžnik, M. Korent, K. Žagar Soderžnik, M. Katter, K. Üstüner, and S. Kobe, "High-coercivity Nd-Fe-B magnets obtained with the electrophoretic deposition of submicron TbF3 followed by the grain-boundary diffusion process," Acta Mater, vol. 115, pp. 278-284, 2016, doi: 10.1016/j.actamat.2016.06.003.

[10] D. Givord, H. S. Li, and J. M. Moreau, "Magnetic properties and crystal structure of $\mathrm{Nd}_{2} \mathrm{Fe}_{14} \mathrm{~B}$," Solid State Commun., vol. 50, no. 6, pp. 497-499, 1984, doi: 10.1016/0038-1098(84)90315-6.

[11] X. Tang, H. Sepehri-Amin, T. Ohkubo, and K. Hono, "Suppression of non-oriented grains in Nd-Fe-B hot-deformed magnets by $\mathrm{Nb}$ doping," Scr. Mater., vol. 147, pp. 108-113, 2018, doi: 10.1016/j.scriptamat.2017.12.032.

[12] Y. H. Hou, Y. L. Huang, Z. W. Liu, D. C. Zeng, S. C. Ma, and Z. C. Zhong, "Hot deformed anisotropic nanocrystalline NdFeB based magnets prepared from spark plasma sintered melt spun powders," Mater. Sci. Eng. B Solid-State Mater. Adv. Technol., vol. 178, no. 15, pp. 990-997, 2013, doi: 10.1016/j.mseb.2013.06.009.

[13] O. Gutfleisch, "Controlling the properties of high energy density permanent magnetic materials by different processing routes," J. Phys. D. Appl. Phys., vol. 33, no. 17, 2000, doi: $10.1088 / 0022-3727 / 33 / 17 / 201$.

[14] R. K. Mishra, E. G. Brewer, and R. W. Lee, "Grain growth and alignment in hot deformed Nd-Fe-B magnets," J. Appl. Phys., vol. 63 , no. 8 , pp. $3528-3530$, 1988, doi: 10.1063/1.340731.

[15] R. W. Lee, "Hot-pressed neodymium-iron-boron magnets," Appl. Phys. Lett., vol. 46, no. 8, pp. 790-791, 1985, doi: 10.1063/1.95884.

[16] R. W. Lee, E. G. Brewer, and N. A. Schaffel, "MAGNETS," IEEE Trans. Magn., vol. M, no. 5, pp. 1958-1963, 1985.

[17] X. Tang et al., "Relationship between the thermal stability of coercivity and the aspect ratio of grains in Nd-Fe-B magnets: experimental and numerical approaches," Acta Mater., 2019, doi: 10.1016/J.ACTAMAT.2019.11.038. 
[18] J. Liu et al., "Grain size dependence of coercivity of hot-deformed Nd-Fe-B anisotropic magnets," Acta Mater., vol. 82, pp. 336-343, 2015, doi: 10.1016/j.actamat.2014.09.021.

[19] X. D. Xu et al., "Comparison of coercivity and squareness in hot-deformed and sintered magnets produced from a Nd-Fe-B-Cu-Ga alloy," Scr. Mater., vol. 160, pp. 9-14, 2019, doi: 10.1016/j.scriptamat.2018.09.028.

[20] H. Sepehri-Amin, Y. Une, T. Ohkubo, K. Hono, and M. Sagawa, "Microstructure of fine-grained Nd-Fe-B sintered magnets with high coercivity," Scr. Mater, vol. 65, no. 5, pp. 396-399, 2011, doi: 10.1016/j.scriptamat.2011.05.006.

[21] J. Liu et al., "Effect of $\mathrm{Nd}$ content on the microstructure and coercivity of hot-deformed Nd-Fe-B permanent magnets," Acta Mater, vol. 61, no. 14, pp. 5387-5399, 2013, doi: 10.1016/j.actamat.2013.05.027.

[22] M. Soderžnik, B. Ambrožič, K. Žagar Soderžnik, and M. Korent, "Limits of grain boundary engineering in nanocrystalline Nd-Fe-B melt-spun ribbons," Mater. Lett., vol. 264, pp. 20-22, 2020, doi: 10.1016/j.matlet.2020.127338.

[23] J. Liu, H. Sepehri-Amin, T. Ohkubo, K. Hioki, A. Hattori, and K. Hono, "Microstructure evolution of hot-deformed Nd-Fe-B anisotropic magnets," J. Appl. Phys., vol. 115, no. 17, pp. 1518, 2014, doi: 10.1063/1.4867960.

[24] M. Soderžnik et al., "Magnetization reversal process of anisotropic hot-deformed magnets observed by magneto-optical Kerr effect microscopy," J. Alloys Compd., vol. 771, pp. 51-59, 2019, doi: 10.1016/j.jallcom.2018.08.231.

[25] L. H. Lewis, Y. Zhu, D. O. Welch, and L. Henderson, "Evidence for reversal by nucleation in REFeB dieupset magnets Evidence for reversal by nucleation in RE-Fe-B die-upset," vol. 6235, no. 1994, pp. 9-12, 2012, doi: 10.1063/1.358291.

[26] H. Sepehri-Amin et al., "Microstructure and temperature dependent of coercivity of hot-deformed Nd-Fe-B magnets diffusion processed with $\mathrm{Pr}-\mathrm{Cu}$ alloy," Acta Mater, vol. 99, pp. 297-306, 2015, doi: 10.1016/j.actamat.2015.08.013.

[27] K. Hioki, T. Takano, and T. Yamamoto, "Influence of Process Conditions on the Magnetic Properties for Hot Deformed Magnets," Denki-Seiko [Electric Furn. Steel], vol. 79, no. 2, pp. 119-125, 2008, doi: 10.4262/denkiseiko.79.119.

[28] M. Korent et al., "Magnetic properties and microstructure evolution of hot-deformed Nd-Fe-B magnets produced by low-pressure spark-plasma sintering," J. Magn. Magn. Mater., vol. 515, no. July, pp. 2-7, 2020, doi: 10.1016/j.jmmm.2020.167279.

[29] J. Trapp and B. Kieback, "Fundamental principles of spark plasma sintering of metals: part I-Joule heating controlled by the evolution of powder resistivity and local current densities," Powder Metall., vol. 62, no. 5, pp. 297-306, 2019, doi: 10.1080/00325899.2019.1653532.

[30] J. W. Carson and B. H. Pittenger, "Advanced Aluminum Powder Metallurgy Alloys and Composites," ASM Handb. Vol. 7 Powder Met. Technol. Appl., vol. 7, pp. 287-301, 1998, doi: $10.1361 /$ asmhba00015.

[31] Z. H. Zhang, Z. F. Liu, J. F. Lu, X. B. Shen, F. C. Wang, and Y. D. Wang, "The sintering mechanism in spark plasma sintering Proof of the occurrence of spark discharge," Scr. Mater, vol. 81, pp. 56-59, 2014, doi: 10.1016/j.scriptamat.2014.03.011.
[32] N. Chawake, L. D. Pinto, A. K. Srivastav, K. Akkiraju, and B. S Murty, "On Joule heating during spark plasma sintering of metal powders," Scr. Mater., vol. 93, pp. 52-55, 2014, doi: 10.1016/j.scriptamat.2014.09.003.

[33] X. Q. Li, L. Li, K. Hu, Z. C. Chen, S. G. Qu, and C. Yang, "Microstructure and magnetic properties of anisotropic Nd-Fe-B magnets prepared by spark plasma sintering and hot deformation," Trans. Nonferrous Met. Soc. China (English Ed., vol. 24, no. 10, pp. 3142-3151, 2014, doi: 10.1016/S1003-6326(14)63453-1.

[34] T. Tomše et al., "A spark-plasma-sintering approach to the manufacture of anisotropic Nd-Fe-B permanent magnets," $J$. Magn. Magn. Mater, vol. 502, no. October 2019, 2020, doi: 10.1016/j.jmmm.2020.166504.

[35] Y. Zhang et al., "Squareness factors of demagnetization curves for multi-main-phase Nd-Ce-Fe-B magnets with different $\mathrm{Ce}$ contents," J. Magn. Magn. Mater., vol. 487, no. May, 2019, doi: 10.1016/j.jmmm.2019.165355.

[36] S. Liu, N. H. Kang, L. Feng, S. H. Lee, J. H. Yu, and J. G. Lee, "Anisotropic Nanocrystalline Nd-Fe-B-Based Magnets Produced by Spark Plasma Sintering Technique," IEEE Trans. Magn., vol. 51, no. 11, pp. 18-21, 2015, doi: 10.1109/TMAG.2015.2439672.

[37] Z. H. Hu, J. Li, L. H. Chu, and Y. Liu, "Effect of hot deformation temperature on the magnetic and mechanical properties of $\mathrm{NdFeB}$ magnets prepared by spark plasma sintering," J. Magn. Magn. Mater., vol. 323, no. 1, pp. 104-107, 2011, doi: 10.1016/j.jmmm.2010.08.040.

[38] X. Tang, H. Sepehri-Amin, T. Ohkubo, K. Hioki, A. Hattori, and K. Hono, "Coercivities of hot-deformed magnets processed from amorphous and nanocrystalline precursors," Acta Mater., vol. 123, pp. 1-10, 2017, doi: 10.1016/j.actamat.2016.10.026.

[39] F. Wang, W. Shen, J. Fan, J. Du, K. Chen, and J. P. Liu, "Strong texture in nanograin bulk Nd-Fe-B magnets via slow plastic deformation at low temperatures," Nanoscale, vol. 11, no. 13, pp. 6062-6071, 2019, doi: 10.1039/C9NR00107G.

[40] M. Zhu and W. Li, "Texture formation mechanism and constitutive equation for anisotropic thermorheological rare-earth permanent magnets," AIP Adv., vol. 7, no. 5, 2017, doi: $10.1063 / 1.4978700$.

[41] L. Li and C. D. Graham, "The Origin of Crystallographic Texture Produced During Hot Deformation in Rapidly-Quenched NdFeB Permanent Magnets," IEEE Trans. Magn., vol. 28, no. 5, pp. 2130-2132, 1992, doi: $10.1109 / 20.179419$.

[42] M. Leonowicz and H. A. Davies, "Effect of Nd content on induced anisotropy in hot deformed FeNdB magnets," Mater. Lett., vol. 19, no. 5-6, pp. 275-279, 1994, doi: 10.1016/0167-577X(94)90170-8.

[43] E. Castle, R. Sheridan, W. Zhou, S. Grasso, A. Walton, and M. J. Reece, "High coercivity, anisotropic, heavy rare earth-free Nd-Fe-B by Flash Spark Plasma Sintering," Sci. Rep., vol. 7, no. 1, pp. 1-12, 2017, doi: 10.1038/s41598-017-11660-9.

[44] H. R. Cha, K. W. Jeon, J. H. Yu, H. W. Kwon, Y. Do Kim, and J. G. Lee, "Coercivity enhancement of hot-deformed Nd-Fe-B magnet by grain boundary diffusion process using the reaction of NdHxand Cu nanopowders," J. Alloys Compd., vol. 693, pp. 744-748, 2017, doi: 10.1016/j.jallcom.2016.09.167. 
[45] W. Yin, R. Chen, X. Tang, J. Ju, and A. Yan, "Effect of pressless heat treatment on the magnetic performance and microstructure of hot-deformed Nd-Fe-B magnet," J. Magn. Magn. Mater., vol. 482, no. September 2018, pp. 9-13, 2019, doi: 10.1016/j.jmmm.2019.03.045.
[46] E. A. Périgo, H. Takiishi, C. C. Motta, and R. N. Faria, "Microstructure and squareness factor: A quantitative correlation in $(\mathrm{Nd}, \mathrm{Pr}) \mathrm{FeB}$ sintered magnets," J. Appl. Phys., vol. 102, no. 11, 2007, doi: 10.1063/1.2821756. 